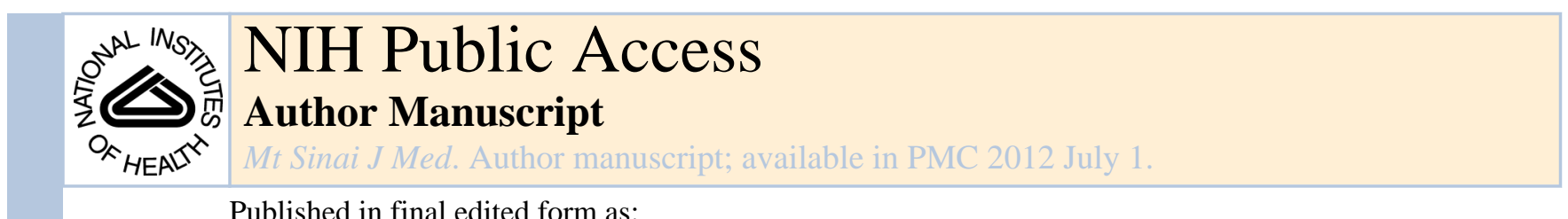

Published in final edited form as:

Mt Sinai J Med. 2011 July ; 78(4): 571-582. doi:10.1002/msj.20267.

\title{
DELIRIUM IN OLDER ADULTS
}

\author{
Dennis M. Popeo, MD \\ Mount Sinai School of Medicine
}

\begin{abstract}
Delirium is a common neuropsychiatric syndrome in the elderly that can occur in several different settings caused by several different processes. It is common and causes increased morbidity and mortality to those affected. This clinical review discusses the prediction, prevention, diagnosis and treatment of delirium in the elderly population. Several strategies to predict delirium are noted with the discussion of pharmacological and non-pharmacological trials of prevention and treatment. Diagnosis of delirium, specifically with the use of objective instruments, is discussed, as is the evidence for pharmacological and non-pharmacological treatment strategies. Discussion of the neurobiology and genetic markers for delirium may elucidate further areas for future research.
\end{abstract}

\section{Keywords}

delirium; older adults

\begin{abstract}
Delirium is a common neuropsychiatric syndrome in the elderly. The DSM-IV-TR defines delirium as a "disturbance of consciousness and a change in cognition that develop over a short period of time." The syndrome includes fluctuations in consciousness over the course of the day, a reduced ability to focus, sustain and shift attention, and evidence that the disturbance is caused by the direct physiological consequence of a medical condition (1).

In general, the prevalence of delirium is dependent on several factors, including the patient population, care setting, the method of study and diversity of antecedent events. The prevalence of delirium in the community is $1-2 \%$, but increases in the setting of general hospital admissions to $6-56 \%$ (2), with the higher prevalence associated with increased age (3) and increased severity of medical illness (4). In the long-term care setting, prevalence of delirium has been noted to fall between 3.4-33.3\% (5). Increased prevalence is noted in areas of advanced levels of care, with $32 \%$ of patients afflicted in general intensive care units (6) and $42 \%$ in cardiac intensive care units (7). Post operative prevalence of delirium in elderly patients ranges from $9-87 \%$, with patients undergoing major, elective, non-cardiac surgeries having a lower prevalence (9\%) of postoperative delirium compared to elderly patients undergoing other surgeries (8). A meta-analysis pooling data from more than 20 studies found that patients (both those who are cognitively intact and those who are impaired) undergoing orthopedic repair after hip fracture have a higher incidence of delirium (21.7\%) than patients undergoing elective surgery (12.1\%) (9). Cardiac surgery has a greater incidence of postoperative delirium when compared with general elective procedures. More complex cardiac procedures, such as valve replacement, have a higher incidence of postoperative delirium than less complex procedures, such as bypass surgeries
\end{abstract}

Correspondence: Dennis.popeo@mssm.edu; (o): 212.659.8865; (m) 646.584.7109; (f) 212.849.2455.

Postmail address Dennis Popeo, MD, 1 Gustave L Levy Place, Box 1230, New York, NY 10029 
(10). Some variability in the prevalence noted in various studies might also be attributed to the method of diagnosis as there are several delirium rating scales and methods of diagnosis.

[Callout] In general, the prevalence of delirium is dependent on several factors, including the patient population, care setting, the method of study and diversity of antecedent events.

The recognition, treatment, outcomes and possible prevention of delirium, postoperative and otherwise, has been the focus of countless papers, making it a topic of clear importance. A recent meta-analysis reviewed more than 2900 articles and found 51 which were of high quality and relevant to calculate the risk of mortality, institutionalization and cognitive impairment in the post-discharge period of elderly patients who were diagnosed with delirium. A mortality calculation involving more than 2000 subjects found an increased risk of death in those diagnosed with delirium when compared to controls at a mean of 11 months (37.2\% vs. $20 \%)$ and a mean of nearly two years (38\% vs. $28.5 \%)$. Similar results were found with the risk of institutionalization for the over 2500 patients included in the analysis at a mean follow up of nearly 15 months (patients with delirium showed a greater risk of institutionalization (33.4\%) than controls (10.7\%)). Moreover, 241 participants in the included studies showed an increased risk of dementia when compared to controls $(62.5 \%$ vs. $8.1 \%)(11)$.

The above meta-analysis is representative of the adverse patient outcomes associated with delirium, but delirium also affects other aspects of the health care system. Delirious patients stay in the hospital longer, accrue greater pharmacy costs and greater professional costs; delirious patients tend to take more medications and require more consultations than their non-delirious counterparts (12). Reducing the length of stay of a delirious patient in the hospital by a single day can amount to $\$ 1-2$ billion in cost savings annually (13). It has been reported that delirium in postoperative setting has been associated with functional impairment, resulting in a severe increase in the loss of independence in performing activities of daily living (14); such that patients are more likely to leave the workforce and more likely to require reliance on disability payments or other social medical/financial safety nets (15). As delirium is common and costly both to the patient and the health care system, it is vital for doctors and hospital staff to predict, prevent, diagnose and treat patients with this condition.

[Callout] As delirium is common and costly both to the patient and the health care system, it is vital for doctors and hospital staff to predict, prevent, diagnose and treat patients with this condition.

\section{PREDICTING DELIRIUM}

Various schema for predicting delirium and elucidating risk factors in different populations of patients have been reported. The majority of the literature focuses on predicting delirium prior to surgical procedures or medical events. A retrospective study of patients undergoing spinal surgery showed that several factors associated with in increased incidence of postoperative delirium: prior central nervous system disorder, advanced age, prior surgeries, history of diabetes mellitus, intraoperative blood transfusion of $800 \mathrm{ml}$ or greater and hematocrit $<100 \mathrm{~g} / \mathrm{L}$ (16). A prospective study of 112 patients admitted to a cardiac surgical intensive care unit showed that increased age and increased duration of surgery were significantly and independently associated with postoperative delirium. Gender, body mass index, presence of diabetes mellitus, preoperative ejection fraction, surgery type, length of cardiopulmonary bypass, intraoperative blood component administration, Acute Physiology and Chronic Health Evaluation II score, Sequential Organ Failure Assessment score, and Charlson Co-morbidity Index were not independently associated with postoperative delirium in this population studied (17). In a small prospective study of 20 cognitively intact, elderly 
patients undergoing elective abdominal surgery, the authors found that increased age, preoperative cognitive dysfunction and lower regional cerebral oxygen saturation were associated with higher instances of post-operative delirium (18). In a study of 119 consecutive cardiac surgical patients who received a brain MRI to assess white matter abnormalities and the relationship of that pathology to post-operative delirium, researchers found that patients with a greater degree of white matter changes had an increased incidence of postoperative delirium (19). In general, patient-specific risk factors such as age, preexisting cognitive and/or functional impairment and greater medical co-morbidities place surgical patients at a higher risk for delirium. When coupled with procedure specific risk factors including degree of operative stress and length of procedure, the risk of delirium increases (20). Rudolph et al elucidated and validated a prediction rule for delirium after cardiac surgery which included impaired cognition, prior stroke or TIA, symptoms of depression and an abnormal level of serum albumin (21). A similar prediction rule was created and validated for patients undergoing elective, non-cardiac surgery. That rule suggested that a higher risk was afforded to those with age over 70, a history of alcohol abuse, cognitive and functional impairment, abnormal electrolytes (specifically sodium, potassium or glucose) and type of surgery, with a higher risk assigned to those undergoing thoracic surgery or aortic aneurysm surgery (22). Thus, any combination of the above risk factors (compiled from the above studies and listed in Table 1 would warrant a high degree of vigilance for the signs and symptoms of delirium, and might suggest that preventive measures should be put into place in the peri-operative setting.

Predictive factors of delirium in non-surgical hospitalized patients have also been elucidated. In a study of consecutive patients admitted to the cardiac intensive care unit at a university hospital with acute myocardial infarctions the 212 who developed delirium were found to have three independently related predictive factors: older age, higher serum potassium at admission, and cardiac arrest during myocardial infarction (23). Inouye and Charpentier developed a predictive model for delirium based on various events / factors during hospitalization. The authors performed two studies to develop and validate a predictive model. The development study was a prospective study that included more than 300 patients admitted consecutively to non-intensive, general medicine floors in a large urban teaching hospital over a 7 month period. Patients were comprehensively evaluated and 25 variables were considered as precipitating factors for delirium. Those factors were narrowed to 5: use of physical restraints, malnutrition, more than 3 medications added in the hospital setting, use of a bladder catheter and any iatrogenic adverse event. In the second study, those variables were validated, and found to predict delirium in patients. When patients were stratified into low, medium or high risks groups by the number of precipitating factors present, patients in higher risk categories had a higher incidence of delirium (24). Inouye later produced a set of predisposing and precipitating factors for delirium in hospitalized patients. A study of more than 280 patients showed that the predisposing factors of vision impairment, severe illness, cognitive impairment and a BUN/Cr ratio greater than or equal to 18 can identify older patients who are at a higher risk for delirium. In a similarly constructed study, precipitating factors that had been previously elucidated were studied with the above predisposing factors. The predisposing and precipitating factors predicted delirium both independently and cumulatively (25). The risk factors that Inouye described are summarized in Table 2.

\section{PREVENTING DELIRIUM}

One strategy to prevent delirium would be to minimize some of the modifiable predicting factors. Minimizing physical restraints, bladder catheters and iatrogenic events, along with maintaining appropriate nutrition should prevent the development of delirium in many patients. The Hospital Elder Life Program (HELP), created at Yale - New Haven Hospital to 
decrease delirium in elderly patients, is an intervention built upon studies of previous attempts at prevention, and combines them with skilled staff members to implement the program throughout the hospital. HELP includes interventions that were tracked for adherence, included activities to increase orientation, early mobilization, aids to augment vision and hearing impairments, sleep enhancement and feeding and fluid assistance. The program also includes specialized assessment with geriatric nurses, interdisciplinary rounds/ consultation, provider education programs, and follow-up after hospital discharge. The interventions were carried out by specially trained nurse specialists, volunteer coordinators, geriatricians and led by a dedicated program director (26). A controlled clinical trial of HELP performed on more than 850 patients, 70 years old or older who were admitted to a general medicine ward at a large, urban teaching hospital. Patients were divided into control (usual care) and intervention groups. The intervention targeted cognitive impairment, sleep deprivation, immobility, visual impairment, hearing impairment and dehydration. Interventions were standardized with specific outcomes that were expected upon reassessment. For instance, in order to modify cognitive impairment, all patients received an orientation protocol at least a once a day; patients were given boards with the names of health care team members, a daily schedule, and thrice daily cognitive stimulation - word games, structured reminiscence, current-events discussions. The outcome that was targeted by the intervention was a change in patients' scores on orientation testing.

Patients in the multifaceted intervention had a significantly lower incidence of delirium than those in the control group. Those in the intervention who suffered delirium suffered significantly fewer days of illness than those in the control group. Patients in this study were also grouped by their risk of delirium. The intervention decreased the incidence of delirium significantly in patients judged to be at an intermediate risk of delirium. Patients in the intervention group that were at a high risk of delirium also had a decreased incidence of delirium when compared to those in the control group, but the results were not significant (27). The intervention cost approximately $\$ 6,300$ per case of delirium prevented. The intervention, in later analysis, was shown to prevent delirium in patients at intermediate risk for delirium without adding to costs, but raised costs in patients judged to be in the high-risk category (28). HELP has been replicated numerous times in community hospitals (29) and other acute care hospitals (30). HELP was not the only multi-component intervention to treat delirium that did not use medications. The results of those studies were variable but did not decrease mortality, effect hospital length of stay, and did not impact post-discharge dependency, function or need for institutional care (31).

Other interventions focus on preventing delirium with prophylactic medications, specifically those medications that are used to treat delirium. Antipsychotic medications are often used to treat delirium, and have been studied for prevention. Haloperidol, a conventional antipsychotic medication, has been studied in several trials. Eighty patients scheduled to undergo gastrointestinal surgery were assigned to either haloperidol or placebo in the perioperative period. Patients in the haloperidol group had a decreased incidence of postoperative delirium (32). Low dose haloperidol was also studied in a randomized, double-blind, placebo-controlled trial to determine the effectiveness of that medication in preventing postoperative delirium in 430 patients who underwent hip surgery. In that study, haloperidol prophylaxis decreased the severity and duration of delirium, and decreased the length of hospital stay for the patients treated. The medication did not reduce the incidence of delirium, however (33). Another study had similar results (34).

Olanzapine, an atypical antipsychotic, has also been tested as a prophylactic agent to prevent postoperative delirium. 495 elderly patients undergoing elective knee or hip replacement were either assigned to a placebo arm or a treatment arm of 10mg of oral olanzapine. The treatment group had a significantly decreased incidence of delirium, but those patients who 
did suffer delirium had a longer, more severe course (35). Although the use of antipsychotic medications can decrease the incidence and severity of delirium, the side effects of these medications, including the increased risk of death, may give clinicians pause before suggesting a more universal use (36).

Donepezil, a cholinesterase inhibitor FDA-approved for the treatment of Alzheimer's disease, has also been tried as a prophylactic agent for delirium. Eighty patients were randomized to receive donepezil 14 days prior to and 14 days after surgery. There was no significant difference in the two groups (37). Another study of donepezil also failed to yield a significant decrease in incidence of delirium or length of hospital stay, but did trend in the direction of a decrease in both (38). There is also evidence from a prospective study of more than 1000 consecutive patients undergoing cardiac surgery with cardiopulmonary bypass over the course of 14 months in an academic hospital. After a review of the data, preoperative administrations of statins had a protective effect, reducing the odds of delirium by $46 \%$ (39). This finding, however, does not fully hold up. In a retrospective chart review of 284,158 consecutive patients age 65 or older who underwent elective surgery, those patients who had taken statins in the perioperative period had a significantly increased risk of postoperative delirium (40).

\section{DIAGNOSING DELIRIUM}

The most important aspect of treating delirium is actually being able to diagnose it. No matter what the skill of a clinician, if delirium is not in the differential, it cannot be diagnosed. Delirium can be diagnosed in any patient who has an acute onset of confusion, inattention, disorganized thinking / behavior, an altered level of consciousness, disturbances of perception or disturbances of sleep/wake cycle. Other conditions often included on the differential diagnosis with delirium include dementia, depression, psychosis, mania and sequelae of neurological conditions (postictal states, aphasia) (41). All of these conditions can be differentiated by the careful taking of a patient's history and the gathering of corroborative information from other sources, including the patient's family members, primary care physicians and other staff members who have cared for the patient.

[Callout] The most important aspect of treating delirium is actually being able to diagnose it.

While making a correct diagnosis of delirium is vital, it is unfortunately, difficult to do. An analysis of data collected from more than 450 patients over the age of 65 treated for femoral neck fractures showed that both physicians and nurses involved in the care of delirious patients did not recognize that the patients were delirious. It was noted that none of the practitioners used scales to help in the diagnosis or delirium (42). One might hypothesize that unit nursing staff, who interact with patients more than physicians, would have a better track record for diagnosing delirium in their patients. They do not. In a study that compared nurse diagnosis of delirium to the observations of a clinical research team, the staff nurses could only recognize delirium in $30.5 \%$ of cases that the research team diagnosed. However, the sensitivity of the nursing diagnosis approached $96 \%$ (43). In this study, as in the previously cited paper, the clinical staff did not use any rating scales to aid in their diagnosis.

A possible problem with arriving at the diagnosis of delirium can be attributed to the differences among its three clinical subtypes: hyperactive, hypoactive and mixed. Hyperactively delirious patients present as restless or agitated and often have hallucinations or delusions. Those with hypoactive delusions are more lethargic and difficult to arouse, with minimal speech and marked psychomotor retardation. Patients with mixed delirium have symptoms of both (44). It is easy to see why some patients, particularly those with a 
hypoactive delirium, might not be diagnosed, as their symptoms are less disruptive to the hospital milieu and do not focus the attention of the staff. However, the symptoms of hypoactive delirium are just as distressing for the patient as a hyperactive or mixed state, and may actually be less responsive to pharmacotherapy (45). Because of the variability in symptoms and the ease with which some symptoms could be missed, a more systematic approach to the diagnosis of delirium, through the use of instruments would seem warranted.

The first such instrument one can utilize to diagnose delirium is the actual DSM criteria. Cameron et al. "field-tested" the use of DSM-III criteria for diagnosing delirium on 133 consecutively admitted patients to the general medical ward of a large urban teaching hospital. Using the criteria, researchers were able to diagnose 20 cases of delirium, 19 more than the clinical staff could on their own. Thus using the DSM criteria for delirium was found to be a reliable screening tool with high inter-rater reliability (46). Others have assessed the utility of DSM-IV-TR criteria for delirium and compared that with ICD-10 criteria and the Memorial Delirium Assessment Scale (MDAS) (47) and found that using the DSM-IV-TR criteria of delirium to screen patients is more inclusive than the ICD-10 criteria (48) (49). The DSM-IV-TR criteria for diagnosing delirium are presented in Table 3.

Many other rating scales for delirium have been developed and may be useful at the bedside, but questions about which instrument is the most valuable remain. Wong et al have tried to shed some light on that question. In a landmark review of bedside delirium instruments, the researchers surveyed more than 6500 citations and focused on 25 studies that met their stringent standards for evidence and inclusion. Those studies included more than 3,000 patients and described the use of 11 different bedside instruments / scales. Likelihood ratios (LR), an indication of how strongly a test result is associated with a disease, were measured for the 11 instruments and 6 of the studied tests had LR greater than 5. Of those 6 scales Global Attentiveness Rating (GAR), Memorial Delirium Assessment Scale (MDAS), Confusion Assessment Method (CAM), Delirium Rating Scale Revised -98 (DRS-R-98), Clinical Assessment of Confusion (CAC) and Delirium Observation Screening Scale (DOSS), - the authors focus on two, the CAM and the GAR, as scales that may be of most use to practitioners. The MMSE, which is often employed for this task due to popularity and familiarity, was not recommended at all because of its low LR (50).

The Global Attentiveness Rating (GAR) rates the patient on the basis of a two-minute conversation between the rater and the patient on general topics. After the conversation, the rater is asked to delineate "how well the patient was able to keep his mind on interacting with you during the interview" and chart this measure on an uninterrupted $10 \mathrm{~cm}$ visual analog scale. This test, which takes relatively little time to give, was significantly correlated with other, established ratings of attentiveness, and showed maximum sensitivity, specificity and positive and negative $\operatorname{LR}(0.94,0.99,94,0.06)$ when used with a cut-off value of 7 (51). This assessment, although simple and time-efficient, was only tested in a small number of patients in one trial, and may not be generalizable or used with clinicians who have less experience with specific populations (50).

The Confusion Assessment Method (CAM) (presented in Table 4) is also a user-friendly, time-efficient instrument developed and copyrighted by Sharon K Inouye of Yale University School of Medicine. It is a four-element diagnostic algorithm based on the DSM criteria for the diagnosis of delirium: acute onset / fluctuating course, inattention, disorganized thinking and altered level of consciousness. A positive test requires the presence of both of the first two symptoms, and one of the last two. The CAM was tested in two differing clinical sites (outpatient and general medicine wards) at two different urban teaching hospitals (Yale University and University of Chicago). Subjects were rated using the CAM by a geriatrician and by clinical interview by a psychiatrist. The CAM showed high sensitivity and specificity 
(94-100\% and 90-95\%) with high inter-rater reliability and high negative predictive accuracy (90-100\%). The likelihood ratios of the four elements of the test ranged from 4.7 to 63. It was noted that the CAM took less than five minutes to administer and could be completed on subjects with severe delirium (52). The CAM has also been adapted for multiple clinical settings (50) and a modification of the CAM, the CAM-ICU (53), is considered by some to be the most valid for use in the Intensive Care Unit (54). Other review articles have tried to elucidate which delirium rating scales are the best, and the CAM rates highly. Adamis et al notes that while the CAM has been validated and is easy to use, practitioners do require some training to fully utilize it. The authors also note that there are many reasons to choose one scale over the other regardless of validity, including purpose (screening vs. diagnostic), clinical setting (emergency room vs. ICU), properties inherent to the scale (ease of use, administrative time), familiarity of the scale, popularity of the scale and organizational culture (55). Again, incorporation of an assessment for delirium into the armamentarium of the clinician can improve patient care, regardless of the instrument used (56).

[Callout] Incorporation of an assessment for delirium into the armamentarium of the clinician can improve patient care, regardless of the instrument used (56).

Aside from the use of instruments to assess patients clinically, electroencephalography (EEG) has been used to diagnose delirium. Results of slowing, dropout of the posterior dominant rhythm, generalized theta or delta slow-wave activity, and poor organization of background rhythms have been noted in the records of delirious patients. These findings however, may not have a high specificity (57). Thus, EEG may help in determining a diagnosis of delirium, but is not a necessary if the diagnosis can be made clinically. Various neuroimaging modalities have been employed to detect delirium. A review of the literature found few studies, with few subjects, which prevented the authors from drawing conclusions about imaging. In general, the studies reviewed found associations between cortical atrophy, ventricular engagement, white matter changes and delirium, but many confounding variables made conclusions difficult to reach (58).

\section{TREATING DELIRIUM}

After the diagnosis of delirium, one should strive to treat it. First and foremost, the causative factor should be elucidated and corrected. In general, the cause of delirium is a medical illness or close result of an event that goes hand in hand with an illness. (FIGURE 1) Of note, the instigating factor of a delirium may be difficult to ascertain and may have occurred in the past, as delirium may not resolve for several days, weeks or months after the precipitating illness has occurred. In this section, possible treatments for medical illness that cause delirium will not be discussed, as they are outside of the scope of this article. Instead, the focus will be on treatment of the symptoms of the delirium itself. Prevention of delirium, however, is probably the best strategy for treatment (26).

There are two basic interventions for a delirious patient: non-pharmacological and pharmacological. One non-pharmacological treatment of delirium is to minimize some of the modifiable predicting / causative factors. Minimizing physical restraints, bladder catheters and iatrogenic events, along with maintaining appropriate nutrition would help in resolving delirium. Maintaining orientation, decreasing environmental stimuli, increasing mobility, preventing dehydration and monitoring medications can all be successful in decreasing length of delirium (59). Mobility seems to play a role in decreasing delirium, and has been shown to decrease the amount of antipsychotic medications used in delirious patients, a measure of severity of delirium (60). Many interventions, however, have not been rigorously tested (61). Focusing on the implementation of the preventative factors strategies discussed above would seem to be more fruitful than the non-pharmacological interventions 
made after the patient is delirious. Regardless of intervention, an educational program to inform hospital staff, along with an algorithmic treatment protocol with some individualization can be associated with better outcomes (62). Some non-pharmacological interventions to treat delirium are detailed in Table 5.

[Callout] Regardless of intervention, an educational program to inform hospital staff, along with an algorithmic treatment protocol with some individualization can be associated with better outcomes.

Pharmacological interventions are often used in the treatment of delirium but none are FDAapproved. Several classes of medications have been used to treat the symptoms of delirium, including antipsychotic medications (atypical and conventional), cholinesterase inhibitors, antidepressants, benzodiazepines and gabapentin.

Antipsychotic medications are an obvious choice to treat delirium as many of the symptoms of delirium are similar to psychosis. There are numerous reviews that discuss the use of this class of medications in delirium. In one review of 23 trials of atypical antipsychotics in the treatment of delirium, researchers found that antipsychotics were safe and effective in reducing delirium symptomotology (63). This review included many single agent trials, but few randomized, placebo controlled studies, which is representative of the body of evidence. As for the choice of agent, haloperidol was considered the first-line treatment, but new data suggest that atypical antipsychotics are equal in efficacy and tolerability to conventional antipsychotics (64). Data supporting the use of olanzapine and risperidone in delirium are also favorable (65), and both medications are equally effective (66). Quetiapine also has been shown to reduce the severity of delirium when compared to placebo in 42 delirious patients (67). Aripiprazole also has been noted to be useful in the treatment of delirium (68). Again, the side effects of these medications, including the increased risk of death, must be acknowledged and reconciled with the need to treat a delirious patient (36).

Cholinesterase inhibitors also have been tried as treatments for delirium. Donepezil did not show any effect in treating postsurgical delirium in cognitively intact patients undergoing elective orthopedic surgery (37). In a double-blind, placebo-controlled trial of donepezil to control delirium after hip replacement, the medication did not yield a significant decrease in the incidence of delirium, but the outcomes measured did suggest a trend that the medication could help (69). There have been reports that have shown donepezil to help in cases of delirium, decreasing the duration of the syndrome (70) especially when the delirium occurred in a demented individual (71) (71). Rivastigmine has also been tested as an agent to treat delirium. In a pilot study of 527 consecutive stroke patients, the 26 patients that developed delirium and were treated with rivastigmine, showed improvement (73). Another study of 15 delirious patients who received placebo or rivastigmine showed that the medication was a safe and effective treatment for delirium, but the reduction in the duration of symptoms was not significant (74). Another pilot study of rivastigmine was stopped due to deaths (75) (76). A procholinergic medication, citicoline, was also tested to see if it could modify delirium after hip fracture surgery, but failed to do so (77).

Antidepressant medications have been used to treat delirium. Two case series, one with three patients (78) and one with five patients (79), described the successful treatment of delirium with fluvoxamine, as measured by a rapid decrease in symptoms. These medications may be more attractive to physicians because of the more favorable side-effect profile. There are reports that trazodone can rapidly decrease the symptoms of delirium (80) (81). A trial of mianserin (a tetracyclic antidepressant) in elderly delirious patients found the medication to be effective in rapidly decreasing the symptoms of delirium with a minimum of side effects (82). 
Benzodiazepines have been considered useful in treating delirium related to withdrawal, but there is little evidence to suggest their use in delirium not related to withdrawal. A Cochrane review of the subject failed to yield any good evidence to determine the utility of those medications (83). A double-blind trial of lorazepam also failed to show that that medication had use in treating delirium (84). Also, benzodiazepines may play a role in causing or worsening delirium (85). A pilot study of gabapentin for postoperative delirium in 21 delirious patients showed a significant reduction in symptoms when compared to those taking placebo (86). Various strategies for the pharmacological treatment of delirium are reviewed in Table 6 .

\section{UNDERSTANDING DELIRIUM}

Strides have been made in the understanding of how a medical illness (such as an infection) could cause a neuropsychiatric illness such as delirium. Understanding the neurobiology and genetics of delirium can be helpful in predicting, preventing and treating this disorder.

There are several theories about the pathophysiology of delirium which describe disturbances in various aspects of the brain as likely culprits for this reaction to illness. They include neurotransmitter dysregulation, inflammatory processes, physiological stress reactions, and perturbations of the sleep-wake cycle, among others (87).

Of these theories, the "neurotransmitter" theory has a significant amount of evidence and is widely considered the most likely. It holds that delirium is caused by dysregulation of neurotransmitters. Deficits in Acetylcholine (ACh) may contribute to delirium, and that specific neurotransmitter system is vulnerable on many levels. The synthesis of ACh is affected by hypoglycemia, malnutrition and ischemia. In addition, medications can inhibit and compete for synaptic ACh receptors, or by blocking its release into the synapse (88). Other neurotransmitters, gamma-aminobutyric acid, glutamate, serotonin, dopamine and norepinephrine, are thought to be involved with delirium as well. Dopamine, which in excess may cause psychosis, is thought to play an important role in delirium by itself, and in combination with Ach (89). Serotonin, which is involved in mood, wakefulness and cognition, has been linked to delirium both by its abundance and absence. Delirious patients have elevated levels of a serotonin metabolite in their cerebrospinal fluid (indicating high levels of serotonin), and relative deficiencies of tryptophan, a serotonin precursor that has also been linked to delirium (90).

The inflammatory hypothesis of delirium deals with the effects of acute systemic inflammation (a feature of numerous medical conditions associated with delirium) on the brain. Such effects include increased cytokine production and disruption of the blood-brain barrier that lead to functional and structural changes in brain parenchyma. These changes are associated with acute behavioral and cognitive disturbances (91).

The theory of aberrant stress responses causing delirium is based upon the pathologically elevated levels of cortisol that occurs in response to illness. Exaggerated or sustained levels of glucocorticoids like cortisol can perturb normal cognition and behavioral function, and there is evidence that points to a link between dysregulation of cortisol and delirium (92). Sleep deprivation and changes in the sleep-wake cycle are both symptoms and predictors of delirium, but there is some evidence that such changes can also cause delirium. Disruptions in the pathway of melatonin production are thought to be important in the pathogenesis of delirium (93).

Along with theories of the pathophysiology of delirium, strides have been made in identifying genetic factors that influence the occurrence or outcomes of delirium in a particular individual. The APOE gene, and specifically the epsilon 4 allele polymorphism, 
has been shown to predispose delirious individuals with the allele to a longer duration of delirium (94). Other studies have confirmed that the presence of the APOE4 allele does prolong delirium, but does not seem to predict incidence of delirium. Other genetic polymorphisms are present and have been studied in relation to alcohol withdrawal delirium, but their effect on general delirium has yet to be elucidated (95).

[Callout] Regardless of intervention, an educational program to inform hospital staff, along with an algorithmic treatment protocol with some individualization can be associated with better outcomes.

\section{CONCLUSION}

Delirium is a common neuropsychiatric syndrome that causes significant morbidity and mortality to affected individuals and increases health care costs. Although it is common, delirium is not always readily recognized. Recognition has been aided by the systematic use of standardized instruments. Progress has been made in our ability to predict delirium, and both pharmacological and non-pharmacological interventions have proven successful in delirium prevention. Both pharmacological and non-pharmacological treatments for delirium are effective. As we learn more about the neurobiology and genetics of delirium, more approaches to prevention and treatment are likely to present themselves. With continued study, we will find better strategies to improve patient outcomes and decrease the burden of this syndrome.

\section{Acknowledgments}

Disclosures: Dr. Popeo receives salary support from NIH and HRSA grants, and from an award from the John A. Hartford foundation.

\section{REFERENCES}

(1). American Psychiatric Association. Diagnostic and Statistical Manual of mental Disorders. 4th Ed, text rev.. American Psychiatric Association; Washington DC: 2000.

(2). Fong TG, Tulebaev SR, Inouye SK. Delirium in elderly adults: diagnosis, prevention and treatment. Nat Rev Neurol. 2009; 5:210-220. [PubMed: 19347026]

(3). Inouye SK. Predisposing and precipitating factors for delirium in hospitalized older patients. Dement Geriatr Cogn Disord. 1999; 10:393-400. [PubMed: 10473946]

(4). Bucht G, Gustafson Y, Sandberg O. Epidemiology of delirium. Dement Geriatr Cogn Disord. 1999; 10:315-318. [PubMed: 10473930]

(5). McCusker J, Cole MG, Voyer P, et al. Prevalence and incidence of delirium in long-term care. Int J Geraitr Psychiatry. Jan 27.2011 doi: 10.1002/gps.2654. [Epub ahead of print].

(6). Salluh JI, Soares M, Teles JM, et al. Delirium epidemiology in critical care (DECCA): an international study. Critical Care. 2010; 14:R210. [PubMed: 21092264]

(7). Chang Y, Tsai YF, Lin PJ, Chen MC, Liu CY. Prevalence and risk factors for postoperative delirium in a cardiovascular intensive care unit. Am J Crit Care. 2008; 17:567-575. [PubMed: 18978241]

(8). Demeure MJ, Fain MJ. The elderly surgical patient and postoperative delirium. J Am Coll Surg. 2006; 203(5):752-757. [PubMed: 17084339]

(9). Bruce AJ, Ritchie CW, Blizard R, Lai R, Raven P. The incidence of delirium associated with orthopedic surgery: a meta-analytic review. Int Psychogeriatr. Apr; 2007 19(2):197-214. Epub 2006 Sep 14. [PubMed: 16973101]

(10). Sockalingam S, Parekh N, Bogoch II, et al. Delirium in the postoperative cardiac patient: a review. J Card Surg. Nov-Dec; 2005 20(6):560-7. [PubMed: 16309412] 
(11). Witlox J, Eurelings LSM, de Jonghe JFM, Kalisvaart KJ, Eikelenboom P, van Gool WA. Delirium in elderly patients and the risk of postdischarge mortality, institutionalization and dementia. JAMA. 2010; 304(4):443-451. [PubMed: 20664045]

(12). Franco K, Litaker D, Locala J, Bronson D. The cost of delirium in the surgical patient. Psychosomatics. Jan-Feb; 2001 42(1):68-73. [PubMed: 11161124]

(13). Levkoff SE, Besine RW, Wetle T, et al. Acute coonfusional states (delirium) in the hospitalized elderly. Ann Rev Gerontol Geriatr. 1986; 6:1-26. [PubMed: 3113449]

(14). Bickel H, Gradinger R, Kochs E, Förstl H. High risk of cognitive and functional decline after postoperative delirium. A three-year prospective study. Dement Geriatr Cogn Disord. 2008; 26(1):26-31. [PubMed: 18577850]

(15). Deiner S, Silverstein JH. Postoperative delirium and cognitive dysfunction. Br J Anaesth. Dec; 2009 103(Suppl 1):i41-46. [PubMed: 20007989]

(16). Gao R, Yang Z, Li M, Shi ZC, Fu Q. Probable risk factors for postoperative delirium in patients undergoing soinal surgery. Eur Spine J. 2008; 17(11):1531-1537. [PubMed: 18795347]

(17). Afonso A, Scurlock C, Reich D, et al. Predictive model for postoperative delirium in cardiac surgical patients. Semin Cardiothorac Vasc Anesth. Sep; 2010 14(3):212-7. [PubMed: 20647262]

(18). Morimoto Y, Yoshimura M, Utada K, Setoyama K, Natsumoto M, Sakabe T. Prediction of postoperative delirium after abdominal surgery in the elderly. J Anesth. 2009; 23(1):51-6. Epub 2009 Feb 22. DOI: 10.1007/200540-008-0688-1. [PubMed: 19234823]

(19). Shioiri A, Kurumaji A, Takeuchi T, Matsuda H, Arai H, Nishikawa T. White matter abnormalities as a risk factor for postoperative delirium revealed by diffusion tensor imaging. Am J Geriatr Paychiatry. 2010; 18:743-753.

(20). Robinson TN, Eiseman B. Postoperative delirium in the elderly: diagnosis and management. Clinical Interventions in Aging. 2008; 3(2):351-5. [PubMed: 18686756]

(21). Rudolph JL, Jones RN, Levkoff SE, et al. Derivation and validation of a preoperative prediction rule for delirium after cardiac surgery. Circulation. 2009; 119:229-236. [PubMed: 19118253]

(22). Marcantonio ER, Goldman L, Mangione CM, et al. A clinical prediction rule for delirium after elective noncardiac surgery. JAMA. 1994; 271:134-139. [PubMed: 8264068]

(23). Uguz F, Kayrak M, Cicek E, Kayhan F, Ari H, Altunbas G. Delirium following acute myocardial infarction: incidence, clinical profiles and predictors. Perspective in Psychiatric Care. 2010; 46(2):135-142.

(24). Inouye SK, Charpentier PA. Precipitating factors for delirium in hospitalized elderly persons. JAMA. 1996; 275:852-857. [PubMed: 8596223]

(25). Inouye SK. Predisposing and precipitating factors in delirium in hospitalized older patients. Dement Geriatr Cogn Disord. 1999; 10:393-400. [PubMed: 10473946]

(26). Inouye SK, Bogardus ST Jr, Baker DI, Leo-Summers L, Cooney LM Jr. The hospital elder life program: a model of care to prevent cognitive and functional decline in older hospitalized patients. J Am Geritr Soc. 2000; 48(12):1697-1706.

(27). Inouye SK, Bogardus ST Jr, Charpenter PA, et al. A multicomponent intervention to prevent delirium in hospitalized older patients. N Engl J Med. 1999; 340:669-76. [PubMed: 10053175]

(28). Rizzo JA, Bogardus ST Jr, Leo-Summers L, Williams CS, Acampora D, Inouye SK. Multicomponent targetd intervention to prevent delirium in hospitalized older patients: what is the economic value? Med Care. Jul; 2001 39(7):740-52. [PubMed: 11458138]

(29). Rubin FH, Williams JT, Lescisin DA, Mook WJ, Hassan S, Inouye SK. Replicating the hospital elder life program in a community hospital and demonstrating effectiveness using quality improvement methodology. J Am Geriatr Soc. 2006; 54(6):969-974. [PubMed: 16776794]

(30). Inouye SK, Baker DI, Fugal P, Bradley EH. Dissemination of the hospital elder life program: implementation, adaptation, and successes. J Am Geriatr Soc. 2006; 54(10):1492-9. [PubMed: 17038065]

(31). Holroyd-Leduc JM, Khandwala F, Sink KM. How can delirium best b prevented and managed in older patients in hospital? CMAJ. 2009 DOI:10.1503/cmaj.080519. 
(32). Kaneko T, Cai J, Ishikura T, Kobayashi M, Naka T, Kaibara N. Prophylactic consecutive administration of haloperidol can reduce the occurrence of postoperative delirium in gastrointestinal surgery. Yonago Acta Medica. 1999; 42:179-184.

(33). Kalisvaart KJ, de Jonghe JF, Bogaards MJ, et al. Haloperidol prophylaxis for elderly hip-surgery patients at risk for delirium: a randomized placebo-controlled study. J Am Geriatr Soc. 2005; 53(10):1658-66. [PubMed: 16181163]

(34). Schrader SL, Wellik KE, Demaerschalk BM, Caselli RJ, Woodruff BK, Wingerchuk DM. Adjunctive Haldol prophylaxis reduces postoperative delirium severity and duration in at risk elderly patients. Neurologist. 2008; 14(2):134-7. [PubMed: 18332845]

(35). Larsen KA, Kelly SE, Stern TA, et al. Administration of olanzapine to prevent postoperative delirium in elderly joint-replacement patients: a randomized, controlled trial. Psychosomatics. 2010; 51(5):409-18. [PubMed: 20833940]

(36). Chahine LM, Acar D, Chemali Z. The elderly safety imperative and antipsychotic usage. Harv Rev Psychiatry. 2010; 18(3):158-72. [PubMed: 20415632]

(37). Liptzin B, Laki A, Garb JL, Fingeroth R, Krushell R. Donepezil in the prevention and treatment of post-surgical delirium. Am J Geriatri Psychiatry. 2005; 13(12):100-6.

(38). Sampson EL, Raven PR, Ndhlovu PN, et al. A randomized, double blind, placebo-controlled trial of donepezil hydrochloride (Aricept) for reducing the incidence of postoperative delirium after elective total hip replacement. Int J Geriatr Psychiatry. 2007; 22(4):343-9. [PubMed: 17006875]

(39). Katznelson R, Djaiani GN, Borger MD, et al. Preoperative use of statins is associated with reduced early delirium rates after cardiac surgery. Anesthesiology. 2009; 110(1):67-73. [PubMed: 19104172]

(40). Redelmeier DA, Thiruchelvam D, Daneman N. Delirium after elective surgery among elderly patients taking statins. CMAJ. 2008; 197(7):945-52.

(41). Saxena S, Lawley D. Delirium in the elderly: a clinical review. Postgrad Med J. 2009; 85(1006): 405-13. [PubMed: 19633006]

(42). Gustafson Y, Brännström B, Norberg A, Bucht G, Windlad B. Underdiagnosis and poor documentation of acute confusional states in elderly hip fracture patients. J Am Geritr Soc. 1991; 39(8):760-5.

(43). Inouye SK, Foreman MD, Mion LC, Katz KH, Cooney LM Jr. Nurses' recognition of delirium and its symptoms: comparison of nurse and researcher ratings. Arch Intern Med. 2001; 161(20): 2467-73. [PubMed: 11700159]

(44). Meagher DJ, O'Hanlon D, O'Mahony E, Casey PR, Trzepacz PT. Relationship between symptoms and motoric subtype of delirium. J Neuropsychiatry Clin Neurosci. 2000; 12(1):51-6. [PubMed: 10678513]

(45). de Rooij SE, Schuurmans MJ, van der Mast RC, Levi M. Clinical subtypes of delirium and their relevance for daily clinical practice: a systematic review. Int J Geriatr Psychiatry. 2005; 20(7): 609-15. [PubMed: 16021665]

(46). Cameron DJ, Thomas RI, Mulvihill M, Bronheim H. Delirium: a test of the Diagnostic and Statistical Manual III criteria on medical inpatients. J Am Geriatr Soc. Nov; 1987 35(11):100710. [PubMed: 3499457]

(47). Breitbart W, Rosenfeld B, Roth A, Smith MJ, Cohen K, Passik S. The Memorial Delirium Assessment Scale. J Pain Symptom Manage. 1997; 13:128-137. [PubMed: 9114631]

(48). Kazmierski J, Kowman M, Banach M, et al. The use of DSM-IV and ICD-10 criteria and diagnostic scales for delirium among cardiac surgery patients: results from the IPDACS study. $\mathrm{J}$ Neuropsychiatry Clin Neurosci. 2010; 22(4):426-32. [PubMed: 21037128]

(49). Kazmierski J, Kowman M, Banach M, et al. Clinical utility and use of DSM-IV and ICD-10 Criteria and The Memorial Delirium Assessment Scale in establishing a diagnosis of delirium after cardiac surgery. Psychosomatics. 2008; 49(1):73-6. [PubMed: 18212180]

(50). Wong CL, Holroyd-Leduc J, Simel DL, Straus SE. Does this patient have delirium? Value of bedside instruments. JAMA. 2010; 304(7):779-786. [PubMed: 20716741]

(51). O'Keeffe ST, Gosney MA. Assessing attentiveness in older hospital patients: global assessment versus tests of attention. J Am Geriatr Soc. Apr; 1997 45(4):470-3. [PubMed: 9100717] 
(52). Inouye SK, van Dyck CH, Alessi CA, Balkin S, Siegal AP, Horowitz RI. Clarifying confusion: the confusion assessment method. A new method for detection of delirium. Ann Intern Med. 1990; 113(12):941-8. [PubMed: 2240918]

(53). Ely EW, Margolin R, Francis J, et al. Evaluation of delirium in critically ill patients: validation of the Confusion Assessment Method for the Intensive Care Unit (CAM-ICU). Crit Care Med. 2001; 29(7):1370-9. [PubMed: 11445689]

(54). Luetz A, Heymann A, Radtke FM, et al. Different assessment tools for intensive care unit delirium: which score to use? Crit Care Med. 2010; 38(2):409-18. [PubMed: 20029345]

(55). Adamis D, Sharma N, Whelan PJP, Macdonald AJD. Delirium scales: a review of current evidence. Aging \& Mental Health. 2010; 14(5):543-555. [PubMed: 20480420]

(56). Devlin JW, Fong JJ, Fraser GL, Riker RR. Delirium assessment in the critically ill. Intensive Care Med. 2007; 33(6):929-40. [PubMed: 17401550]

(57). Jacobson S, Jerrier H. EEG in delirium. Semin Clin Neuropsychiatry. Apr; 2000 5(2):86-92. [PubMed: 10837097]

(58). Soiza RL, Sharma V, Ferguson K, Shenkin SD, Seymour DG, MacLullich AMJ. Neuroimaging studies of delirium: a systemic review. J Psychosom Res. 2008; 65:239-248. [PubMed: 18707946]

(59). Aguirre E. Delirium and hospitalized older adults: a review of nonpharmacologic treatment. J Contin Educ Nurs. 2010; 41(4):151-2. [PubMed: 20411884]

(60). Tatematsu N, Hayashi A, Narita K, Tamaki A, Tsuboyama T. The effects of exercise therapy on delirium in cancer patients: a retrospective study. Support Care Cancer. 2010 [Epub ahead of print] DOI: 10.1007/s00520-010-0874-1.

(61). Skorbik Y. Delirium prevention and treatment. Crit Care Clin. 2009; 25:585-591. [PubMed: 19576532]

(62). Skrobik Y, Ahern S, Leblanc M, Marquis F, Awissi DK, Kavanagh BP. Protocolized intensive care unit management of analgesia, sedation and delirium improves analgesia and subsyndromal delirium rates. Anesth Analg. 2010; 111:451-63. [PubMed: 20375300]

(63). Peritogiannis V, Stefanou E, Lixouriotis C, Gkogkos C, Rizos DV. Atypical antipsychotics in the treatment of delirium. Psychiatry Clin Neurosci. 2009; 63(5):623-31. [PubMed: 19674385]

(64). Campbell N, Boustani MA, Ayub A, et al. Pharmacological management of delirium in hospitalized adults - a systematic evidence review. J Gen Intern Med. 2009; 24(7):848-53. [PubMed: 19424763]

(65). Attard A, Ranjith G, Taylor D. Delirium and its treatment. CNS Drugs. 2008; 22(8):631-44. [PubMed: 18601302]

(66). Kim SW, Yoo JA, Lee SY, et al. Risperidone versus olanzapine for the treatment of delirium. Hum Psychopharmacol. 2010; 24(4):298-302. [PubMed: 20521319]

(67). Tahir TA, Eeles E, Karapareddy V, et al. A randomized controlled trial of quetiapine versus placebo in the treatment of delirium. J Psychosom Res. 2010; 69(5):485-90. [PubMed: 20955868]

(68). Crocq MA, Camus V, Millet B, et al. Clinical potentialities and perspectives for the use of aripiprazole in other disorders than its classical indications. A critical analysis of the recent literature. Encephale. 2008; 34(2):187-93. [PubMed: 18597728]

(69). Sampson EL, Raven PR, Ndhlovu PN, et al. A randomized, double-blind, placebo-controlled trial of donepezil hydrochloride (Arcept) for reducing the incidence of postoperative delirium after elective total hip replacement. Int J Geriatr Psychiatry. 2007; 22(4):343-9. [PubMed: 17006875]

(70). Gleason OC. Donepezil for postoperative delirium. Psychosomatics. 2003; 44(5):437-8. [PubMed: 12954923]

(71). Wengel SP, Roccaforte WH, Burke WJ. Donezepil improves symptoms of delirium in dementia: implications for future research. J Geraitr Psychiatry Neurol. 1998; 11(3):159-61.

(72). Wengel SP, Burke WJ, Roccaforte WH. Donepezil for postoperative delirium associated with Alzheimer's disease. J Am Geriatr Soc. 1999; 47(3):379-80. [PubMed: 10078910]

(73). Oldenbeuving AW, de Kort PL, Jansen BP, Kapelle J, Roks G. A pilot study of rivastigmine in the treatment of delirium after stroke: a safe alternative. BMC Neurol. 2008; 8:34. [PubMed: 18803862] 
(74). Overshott R, Vernon M, Moris J, Burns A. Rivastigmine in the treatment of delirium in older people: a pilot study. Int Psychogeriatr. 2010; 22(5):812-8. [PubMed: 20353624]

(75). Sheldon T. Study of rivastigmine for delirium in intensive care is stopped after deaths. BMJ. 2010; 340:c2895. [PubMed: 20511318]

(76). Van Eijk MM, Roes KC, Honing ML, et al. Effect of rivastigmine as an adjunct to usual care with haloperidol on duration of delirium and mortality in critically ill patients: a multicentre, double-blind, placebo-controlled randomisied trail. Lancet. 2010; 376(9755):1829-37. [PubMed: 21056464]

(77). Diaz V, Rodriguez J, Barrientos P, et al. Use of procholinergics in the prevention of postoperative delirium in hip fracture surgery in the elderly. A randomized controlled trail. Rev Neurol. 2001; 33(8):716-9. [PubMed: 11784964]

(78). Furuse T, Hashimoto K. Sigma-1 receptor agonist fluvoxamine for postoperative delirium in older adults: report of three cases. Ann Gen Psychiatry. 2010; 9:28. [PubMed: 20573265]

(79). Furuse T, Hashimoto K. Sigma-1 receptor agonist fluvoxamine for postoperative delirium in intensive care units: report of five cases. Ann Gen Psychiatry. 2010; 9:18. [PubMed: 20416097]

(80). Davis MP. Does trazodone have a role in palliating symptoms? Support Care Cancer. 2007; 15(2):221-4. [PubMed: 17131134]

(81). Okamoto Y, Matsuoka Y, Sasaki T, Jitsuili H, Horiguchi J, Yamawaki S. Trazdone in the treatment of delirium. J Clin Psychopharmacol. 1999; 19(3):280-2. [PubMed: 10350040]

(82). Uchiyama M, Tanaka K, Isse K, Toru M. Efficacy of mianserin on symptoms of delirium in the aged: an open trail study. Prog Neuropsychopharmacol Biol Psychiatry. 1996; 20(4):651-6. [PubMed: 8843489]

(83). Lonergan E, Luxenberg J, Areosa Sastre A. Benzodiazepines for delirium. CochraneDatabase Syst Rev. 2009; (4):CD006739.

(84). Breitbart W, Marotta R, Platt MM, et al. A double-blind trial of Haldol, chlorpromazine and lorazepam in the treatment of delirium in hospitalized AIDS patents. Am J Psychiatry. 1996; 153(2):231-7. [PubMed: 8561204]

(85). Alldred DP. Avoid benzodiazepines and opioids in people at risk of delirium. Evidence-Based Nursing. 2011 oi:10.1136/ebn1155.

(86). Leung JM, Sands LP, Rico M, et al. Pilot clinical trial of gabapentin to decrease postoperative delirium in older patients. Neurology. 2006; 67(7):1251-3. [PubMed: 16914695]

(87). Van Munster BC, de Rooij SE, Korevaar JC. The role of genetics in delirium in the elderly patient. Dement Geriatr Cogn Disord. 2009; 28:187-195. [PubMed: 19713702]

(88). Hshieh TT, Fong TG, Marcantonio ER, Inouye SK. Cholinergic deficiency hypothesis in delirium: a synthesis of current evidence. J Gerontol A Biol Sci Med Sci. 2008; 63(7):764-772. [PubMed: 18693233]

(89). Gunther ML, Morandi A, Ely EW. Pathophysiology of delirium in the intensive care unit. Crit Care Clin. 2008; 24:45-65. [PubMed: 18241778]

(90). Flacker JM, Lipsitz LA. Neural mechanisms of delirium: current hypotheses and evolving concepts. J Gerontol A Biol Sci Med Sci. 1999; 54a(6):B239-B246. [PubMed: 10411009]

(91). Cerejeira J, Firmino H, Vaz-Serra A, Mukaetova-Ladinska EB. The neuroinflammatory hypothesis of delirium. Acta Neuropathol. 2010; 119:737-754. [PubMed: 20309566]

(92). MacLullich AMJ, Ferguson KJ, Miller T, de Rooij SEJA, Cunningham C. Unravelling the pathophysiology of delirium: a focus on the role of aberrant stress responses. J Psychosom Res. 2008; 65:229-238. [PubMed: 18707945]

(93). Figueroa-Ramos MI, Arroyo-Novoa CM, Lee KA, Padilla G, Puntillo KA. Sleep and delirium in ICU patients: a review of mechanisms and manifestations. Intensive Care Med. 2009; 35:781795. [PubMed: 19165463]

(94). Ely WE, Girard TD, Shintani AK, et al. Apolipoprotein E4 ploymorphism as a genetic predisposition to delirium in critically ill patients. Crit Care Med. 2007; 35:112-117. [PubMed: 17133176]

(95). Adamis D, Van Munster BC, MacDonald AJD. The genetics of deliria. Int Rev Psychiatry. 2009; 21(1):20-29. [PubMed: 19219710] 


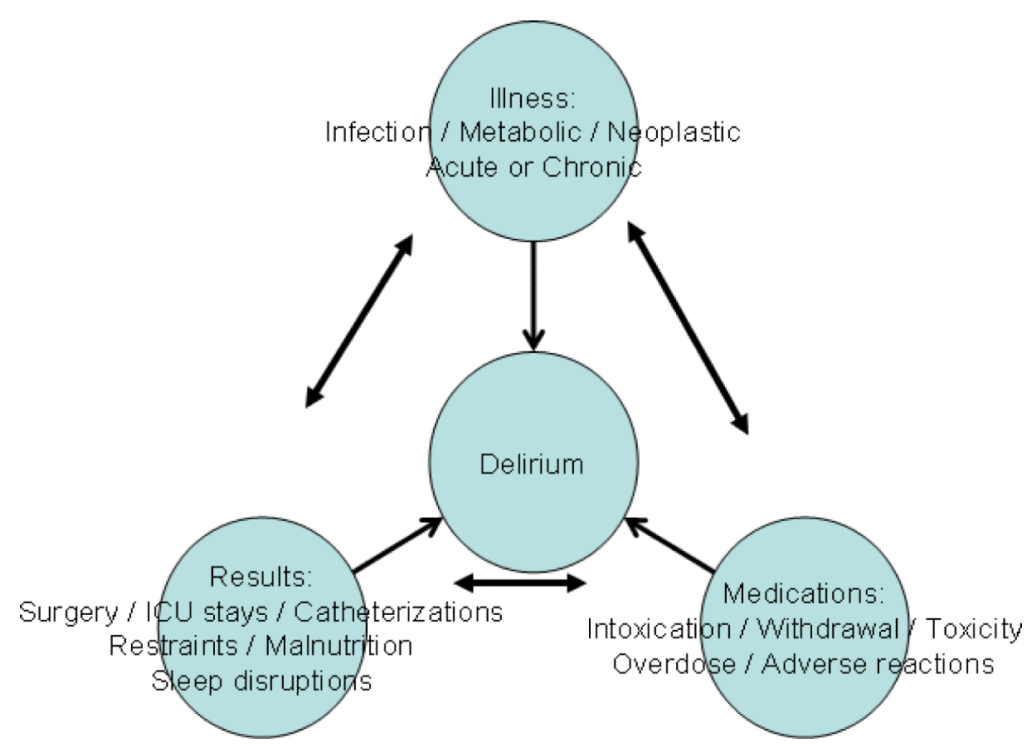

Figure 1.

Illness is the prerequisite for delirium, but the medications used to combat illness and the resulting interventions can also lead to delirium, and interact with each other to cause delirium 
Table 1

Risk Factors for Delirium in Surgical Patients.

Advanced age
Existing cognitive impairment
Existing functional impairment
History of CVA / TIA / CNS disorder
History of alcohol abuse
Metabolic abnormalities: abnormal albumin, sodium, potassium, glucose
Diabetes

Type and complexity of surgery. 
Table 2

Risk Factors for Delirium in Medical Patients.

Vision impairment
Severe illness
Cognitive impairment
BUN/Cr ratio $\geq 18$
Precipitating factors for delirium
Use of physical restraints
Malnutrition
More than 3 medications added during the hospitalization
Use of a bladder catheter
Any iatrogenic event




\section{Table 3}

\section{DSM IV-TR Criteria for Delirium}

A: Disturbance in consciousness with reduced ability to focus, sustain or shift attention.

B: A change in cognition or the development of a perceptual disturbance that is not better accounted for by a preexisting, established or evolving dementia.

C: The disturbance develops over a short period of time and tends to fluctuate over the course of the day.

D: There is evidence from history, physical examination, or laboratory findings that the disturbance is caused by the direct physiological consequences of a general medical condition.

CLINICAL SUBTYPES:

HYPERACTIVE: agitation, restlessness, with hallucinations and /or delusions

HYPOACTIVE: lethargic, difficult to arouse, minimal speech, psychomotor retardation

MIXED: symptoms of both hyperactive and hypoactive delirium 


\section{Table 4}

The Confusion Assessment Method (CAM) Diagnostic Algorithm.

Copyright owned by Sharon K. Inouye, Yale University School of Medicine

The diagnosis of delirium requires the presence/abnormal rating for features 1 and 2 and also either features 3 or 4 .

Feature 1: Acute onset and fluctuating course

Is there an acute change in mental status / cognition and does this characteristic fluctuate over the course of the day?

Feature 2: Inattention

Does the patient have difficulty focusing attention?

Feature 3: Disorganized thinking

Is the patient's speech disorganized?

Feature 4: Altered level of consciousness

How would you describe the patient's consciousness?

Normal/Alert; Vigilant/Hyperalert; Lethargic; Stupor; Coma 
Table 5

Non-pharmacological Treatments of Delirium

Minimize use of physical restraints

Minimize use of bladder catheters

Minimize iatrogenic events

Maintain appropriate nutrition

Maintain orientation

Decrease environmental stimuli

Increase mobility earlier

Prevent dehydration

Monitor medications for side effects that can increase delirium

Implement educational program to inform hospital staff and an algorithmic treatment protocol 
Table 6

\section{Pharmacologicl Treatments of Delirium}

Antipsychotic Medications - both conventional and atypical antipsychotics are effective in treating delirium and have been studied in randomized, placebo controlled trials. Atypical antipsychotics tend to have fewer side effects, but both classes of medications carry black box warnings about their use in the elderly.

Cholinesterase Inhibitors - Case reports and trials of this class of medications have shown that there may be a role for their use. Side effects, and the halt of one trial because of patient deaths make their use less palatable. Procolinergic medications have failed to reduce symptoms of delirium.

Antidepressants - Case reports show that these medications, specifically SSRIs, TCAs and trazodone can be useful in reducing symptoms of delirium.

Benzodiazepines - No real use in treating delirium that is not due to withdrawal. 